\title{
Adaptive Dynamics of Speciation: Spatial Structure
}

Michael Doebeli (doebeli@zoology.ubc.ca)

Ulf Dieckmann (dieckman@iiasa.ac.at)

\section{Approved by}

Leen Hordijk (hordijk@iiasa.ac.at)

Director, IIASA

November 2004 


\section{Contents}

1 Introduction 1

2 Classic Models of Parapatric Speciation 2

3 Evolutionary Branching in Spatially Structured Populations 3

4 Extension to Sexual Populations: Parapatric Speciation 14

5 A Note on Species-Area Relationships 19

6 Concluding Comments 21 


\author{
About the Authors \\ Michael Doebeli \\ Department of Zoology \\ University of British Columbia \\ 6270 University Blvd. \\ Vancouver BC V6T 1Z4, Canada \\ Ulf Dieckmann \\ Adaptive Dynamics Network \\ International Institute for Applied Systems Analysis \\ A-2361 Laxenburg, Austria
}

\title{
Acknowledgments
}

Michael Doebeli gratefully acknowledges financial support from the National Science and Engineering Council (NSERC) of Canada, and from the James S. McDonnell Foundation, USA.

Ulf Dieckmann gratefully acknowledges financial support from the Austrian Science Fund, from the Austrian Federal Ministry of Education, Science, and Cultural Affairs, and from the European Research Training Network ModLife (Modern Life-History Theory and its Application to the Management of Natural Resources), funded through the Human Potential Programme of the European Commission. 


\title{
Adaptive Dynamics of Speciation: Spatial Structure
}

\author{
Michael Doebeli \\ Ulf Dieckmann
}

\section{Introduction}

Extant patterns of species abundance are usually considered to be suggestive of allopatric speciation, because even closely related species are often geographically segregated (e.g., Barraclough and Vogler 2000; see Chapters 15, 16, and 17 in Dieckmann et al. 2004). Even though, in many cases, the ecological abutment between related species does not correspond to any obvious geographic barriers to gene flow, such patterns of geographic segregation are taken as strong indicators that speciation has occurred, either in allopatry or in parapatry. For the latter case it is assumed implicitly that there exists some sort of environmental discontinuity on either side of which different types are favored by selection or have evolved by genetic drift (Turelli et al. 2001). Even though gene flow across the environmental discontinuity can actually enhance speciation through the process of reinforcement, in these parapatric scenarios the reasons for speciation are ultimately the same as those in purely allopatric scenarios, that is, divergent evolution in different geographic regions. This has led to a common understanding that allopatric patterns of abundance between closely related species imply past events of allopatric speciation.

Rather than focusing on patterns of species abundance, recent developments in speciation theory focused on the adaptive processes and mechanisms that lead to disruptive selection and subsequent divergence of emerging new lineages in well-mixed, geographically unstructured populations. While this approach, described in Chapters 4 and 5 in Dieckmann et al. 2004, highlights the importance of frequency-dependent ecological interactions for evolutionary diversification, nonspatial models evidently cannot explain geographic patterns of species abundance. For this it is necessary to account for spatial structure explicitly, including spatial heterogeneity in environmental conditions and spatially localized ecological interactions.

Most previous studies of the role of spatial structure for evolutionary diversification assumed a discrete spatial population subdivision into local habitat patches within which interactions were unstructured spatially (e.g., Chapter 3 in Dieckmann et al. 2004; Boxes 4.5 and 4.6 in Chapter 4 in Dieckmann et al. 2004; Day 2000). This chapter describes how the study of evolutionary branching as a model for adaptive speciation can be extended to spatially structured populations that occupy a continuous spatial area. The resultant models of spatial evolutionary branching establish a firm link between ecological processes and geographic patterns of speciation. An abbreviated version of this theory can be found in Doebeli and Dieckmann (2003).

Adhering to established terminology, the models described below are models of parapatric speciation, because interactions between individuals only occur over short spatial distances and the populations are not panmictic. At the same time, however, these models differ crucially from traditional models of parapatric speciation in that the disruptive selective forces responsible for speciation are not imposed externally by the environment, but instead emerge dynamically from local adaptation in conjunction with spatially localized ecological interactions. Specifically, in contrast to the environmental discontinuities assumed, for example, in traditional models for stepped 
genetic clines in hybrid zones (Barton and Hewitt 1989), we assume that environmental conditions change gradually over space along a linear environmental gradient. If evolution were solely driven by adaptation to this gradient and limited only by gene flow, the establishment of a corresponding phenotypic gradient over space would be expected (Slatkin 1978; Kirkpatrick and Barton 1997; Barton 1999). A crucial perspective that the results described below add to this simple picture is that local adaptation along an environmental gradient has the potential to increase the strength of frequency-dependent selection in the system: if local adaptation leads to a correlation between phenotype and spatial location, and if interactions are spatially localized, then individuals tend to interact relatively more often with other individuals of similar phenotypes. As we show later, this mechanism actually greatly facilitates evolutionary branching in spatially structured populations. Interestingly, this facilitation is most pronounced for environmental gradients of intermediate slope. Moreover, when evolutionary branching occurs, the newly emerging lineages are often spatially segregated, and show a pattern of species abutment. In this way, the models for parapatric speciation studied here link local processes that drive evolutionary diversification to global patterns of species abundance. These results show that, contrary to the predictions of traditional parapatric models, ecological contact is not necessarily a hindrance to speciation, but, instead, can be a prerequisite for speciation.

Section 2 briefly reviews traditional approaches to parapatric speciation. We then present individual-based models for evolutionary branching in spatially extended populations, first for clonally reproducing individuals (Section 3) and second for sexual populations in which assortative mating must evolve for speciation to occur through evolutionary branching (Section 4). Finally, in Section 5 we point out a potential link between adaptive speciation in spatially structured populations and the origin of species-area relationships: for a given resource diversity along an environmental gradient, conditions for adaptive speciation are less restrictive in larger spatial areas.

\section{Classic Models of Parapatric Speciation}

Parapatric speciation occurs when an ancestral population splits into divergent descendant lineages that occupy different geographic areas in the ancestral species range under the maintenance of at last some gene flow across the spatial boundaries between the emerging species. As for the theoretical plausibility of this scenario, it is agreed widely that "any mechanism that can produce divergence among allopatric populations can also cause divergence in parapatry" (Turelli et al. 2001, p. 337). Parapatric speciation is thus generally envisaged as a process in which the divergence of types occurs in different geographic regions of an ancestral species range, accompanied or followed by the emergence of reproductive isolation between the diverging lineages. Divergence may result from selection or drift, and reproductive isolation between diverging subpopulations may be a pleiotropic by-product of local adaptation, as in allopatric speciation, or it may be an adaptive consequence of reinforcement (see Turelli et al. 2001 for a review).

Situations in which divergence is caused by genetic drift are described in detail in Chapter 6 in Dieckmann et al. 2004. In such cases it is usually assumed that reproductive isolation occurs automatically (through pleiotropic side-effects) as soon as the genetic distance between local populations is large enough (Gavrilets 1999). By contrast, when divergence is caused by local adaptation, reproductive isolation can arise either pleiotropically or through reinforcement (i.e., through the evolution of mating barriers between locally adapted populations driven by selection against hybrids). The latter mechanism (which generates a speciation process that is partially adaptive; see Chapter 19 in Dieckmann et al. 2004) has received considerable attention in the theoretical literature [Liou and Price 1994; Noor 1995; Kirkpatrick and Servedio 1999; see also the comprehensive review by Turelli et al. (2001)]. Even though details of genetic architecture, population structure, 
hybrid inferiority, and mating systems differ between the various studies, the general conclusion from these studies is that reinforcement is a theoretically plausible evolutionary scenario (Turelli et al. 2001).

While the evolution of traits that influence prezygotic isolation in the presence of selection against hybrids is naturally at center stage in studies of parapatric speciation through reinforcement, the ecological reasons as to why hybrid inferiority exists in the first place have received less attention. Typically, it is assumed tacitly that the ecological divergence, which leads to the establishment of different local types and to hybrid inferiority in the contact zone, is caused by externally given discontinuities in the environment (e.g., by the existence of local habitats with different adaptive peaks within a species' range, or by stepped environmental clines). Indeed, reinforcement classically refers to evolutionary processes that unfold upon secondary contact between populations for which ecological divergence has occurred in allopatry. Even though the same process can, in principle, occur during primary contact (i.e., under conditions of continual gene flow), the underlying environmental discontinuities necessary for local adaptation and hybrid inferiority must then be assumed a priori.

A notable exception to this conventional pattern are the models by Endler (1977) for parapatric speciation along clines. In these models, the fitness of different genotypes can vary linearly, rather than stepwise, along environmental gradients, and yet stepped genotypic clines can occur. These steps are, however, contingent on the special genetic architecture considered: with one locus and two alleles determining fitness, there simply exists a point along the environmental gradient at which the relative fitness of the two alleles changes sign. It is therefore easy to show that Endler's stepped genotypic clines disappear when more loci or alleles are allowed to affect fitness.

In contrast to these traditional approaches to parapatric speciation, here we focus on the intrinsic ecological mechanisms that can generate divergence and stepped phenotypic clines along continuous environmental gradients. To understand the underlying adaptive processes of divergence, we first discuss clonal models, in which reproductive isolation is not an issue. We then extend these models to sexual populations, in which reinforcement during primary contact can lead to the evolution of assortative mating and hence allow for speciation.

\section{Evolutionary Branching in Spatially Structured Populations}

Whatever the mechanism of adaptive speciation, some form of disruptive selection must be involved. Recent advances in the theory of adaptive dynamics (Metz et al. 1996; Geritz et al. 1998; Doebeli and Dieckmann 2000) demonstrate that disruptive selection regimes caused by frequencydependent interactions emerge dynamically during the evolutionary process in generic models for all basic types of ecological interactions, a finding that was foreshadowed in earlier work by Eshel (1983), Christiansen (1991), and Abrams et al. (1993a). Such disruptive selection can lead to evolutionary branching, that is, to the splitting of evolving lineages into two phenotypic clusters (Chapters 4 and 5 in Dieckmann et al. 2004). Here we extend the study of evolutionary branching to spatially structured models for resource competition so as to investigate the effect of localized interactions in geographically extended populations on adaptive speciation along environmental gradients. We first focus on the simpler case of asexual populations to reveal ecological settings that are speciation prone.

Our starting point is the generic Lotka-Volterra models for frequency-dependent competition in spatially unstructured populations, which are introduced in Chapter 5 in Dieckmann et al. 2004, and which we briefly recall here. In these models, individuals vary with respect to a quantitative trait $x$, which could be a morphological, behavioral or physiological character. The deterministic 
dynamics of the density $n_{x}$ of a population that is monomorphic for trait value $x$ is given by

$$
\frac{\mathrm{d} n_{x}}{\mathrm{~d} t}=r n_{x}\left[1-n_{x} / K(x)\right]
$$

where $r$ is the intrinsic growth rate of the species, which is assumed to be independent of the phenotype $x$. The carrying capacity $K(x)$ is the equilibrium density of populations that consist of $x$ individuals and reflects the abundance of resources available to such individuals. We assume $K(x)$ to be of normal form,

$$
K(x)=K_{0} \exp \left(-\frac{1}{2}\left(x-x_{0}\right)^{2} / \sigma_{K x}^{2}\right) .
$$

This implies that some intermediate phenotype $x_{0}$ has maximal carrying capacity, and that the decline of $K(x)$ to either side of $x_{0}$ is measured by the standard deviation $\sigma_{K x}$ of the normal distribution $z$. The equilibrium population density $\hat{n}_{x}=K(x)$ of a population monomorphic for $x$ is asymptotically stable. Considering such a resident population at its carrying capacity, the fate of a mutant phenotype $x^{\prime}$ is determined by its per capita growth rate when rare,

$$
f\left(x^{\prime}, x\right)=\frac{\mathrm{d} n_{x^{\prime}}}{n_{x^{\prime}} \mathrm{d} t}=r\left[1-a\left(x^{\prime}, x\right) K(x) / K\left(x^{\prime}\right)\right] .
$$

Here $K\left(x^{\prime}\right)$ is the carrying capacity of the mutant $x^{\prime}$, and $a\left(x^{\prime}, x\right)$ measures the strength of competition exerted by phenotype $x$ on phenotype $x^{\prime}$. This function is assumed to have the form

$$
a\left(x^{\prime}, x\right)=\exp \left(-\frac{1}{2}\left(x^{\prime}-x\right)^{2} / \sigma_{a x}^{2}\right),
$$

which implies that competition is strongest between individuals of similar phenotypes, as would occur, for example, when similarly sized individuals compete for similar types of food. Consequently, the total competition that impinges on an individual depends on the phenotypic composition of the population it is part of. In particular, a rare mutant $x^{\prime}$ pitched against a resident $x$ at carrying capacity $K(x)$ experiences competition from a discounted number of individuals $a\left(x^{\prime}, x\right) K(x)$ for an amount of resources that is proportional to $K\left(x^{\prime}\right)$, a consideration that immediately allows us to understand the mutant's fitness $f\left(x^{\prime}, x\right)$ in Equation (1c).

The adaptive dynamics of the trait $x$ is determined by the selection gradient $g(x)=$ $\partial f\left(x^{\prime}, x\right) /\left.\partial x^{\prime}\right|_{x^{\prime}=x}$. According to the canonical equation of adaptive dynamics (Dieckmann and Law 1996), the rate of mutation-limited evolutionary change $\mathrm{d} x / \mathrm{d} t$ is proportional to $g(x)$, with the constant of proportionality determined by the mutational process that generates genetic variability. The same basic proportionality also arises in models of quantitative genetics that describe evolution in genetically polymorphic populations (Lande 1979b). Using the functional forms of $K(x)$ and of $a\left(x^{\prime}, x\right)$, Equations (1b) and (1d), it is easy to see that the trait value $x_{0}$ that maximizes the carrying capacity is the only trait value for which the selection gradient vanishes, $g\left(x_{0}\right)=0$ (i.e., the only evolutionary equilibrium in phenotype space). The phenotype $x_{0}$ is also a global evolutionary attractor. This is because $\mathrm{d} g / \mathrm{d} x\left(x_{0}\right)<0$, which implies that for resident trait values $x$ smaller than $x_{0}$ selection acts to increase $x$, and for resident trait values larger than $x_{0}$ selection acts to decrease $x$. Thus, independent of the initial trait value considered, evolutionary trajectories converge toward $x_{0}$.

However, $x_{0}$ need not be evolutionarily stable, which means a population monomorphic for $x_{0}$ may be susceptible to invasion by nearby mutants. Evolutionary stability is determined by the second derivative of the fitness function $f\left(x^{\prime}, x\right)$ with respect to $x^{\prime}$, evaluated at $x_{0}$ : if $\partial^{2} f\left(x^{\prime}, x\right) /\left.\partial x^{\prime 2}\right|_{x^{\prime}=x=x_{0}}<0$, then $x_{0}$ is a fitness maximum, and hence evolutionarily stable. In this case $x_{0}$ represents a final stop for the adaptive dynamics.

In contrast, if $\partial^{2} f\left(x^{\prime}, x\right) /\left.\partial x^{\prime 2}\right|_{x^{\prime}=x=x_{0}}>0$, then $x_{0}$ is a fitness minimum, and hence an evolutionary branching point (Chapter 4 in Dieckmann et al. 2004). In this case, the population first 


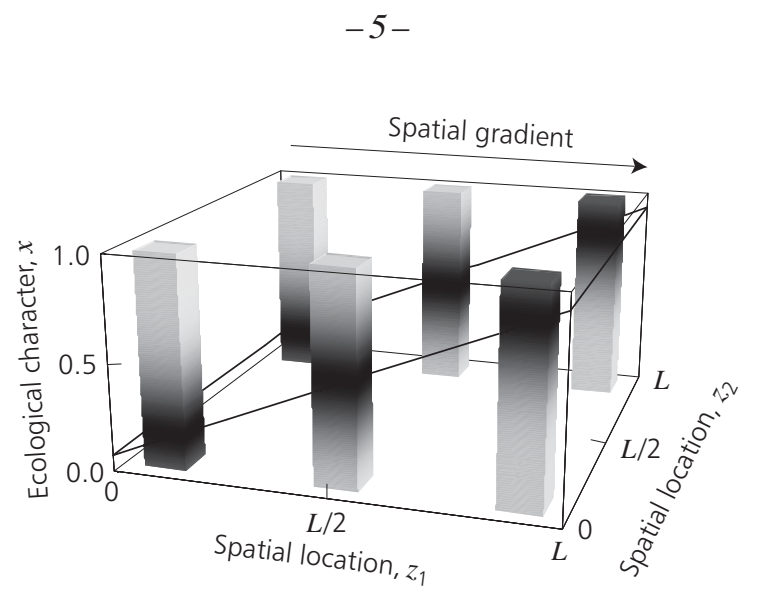

Figure 1 Environmental gradient in carrying capacities. The dark shading corresponds to phenotypes that maximize local carrying capacity; these gradually change with spatial location in the $z_{1}$ direction, while the $z_{2}$ direction is ecologically neutral. At any given location, the carrying capacity decreases with phenotypic distance from the capacity-maximizing phenotype (indicated by diminished darkness).

evolves to $x_{0}$ and then splits into two phenotypic lineages that diverge from $x_{0}$ as well as from each other. For the model investigated here it is established easily that $x_{0}$ is a branching point if $\sigma_{a x}<\sigma_{K x}$, that is, if around $x_{0}$ the strength of competition decreases faster with phenotypic distance than the carrying capacity (Chapter 5 in Dieckmann et al. 2004).

This analytical theory for the adaptive dynamics of resource competition in well-mixed populations serves as a reference point for the spatially structured models introduced below. It can be derived from underlying individual-based stochastic models (Dieckmann and Law 1996). These individual-based models are described in Chapter 5 in Dieckmann et al. 2004; we now extend them to spatially structured populations by making the following assumptions. In addition to its phenotype $x$, each individual is characterized by its spatial location $\left(z_{1}, z_{2}\right)$ in a square of continuous space with sides of length $L$. In this spatial arena, resources are distributed such that for each spatial location $\left(z_{1}, z_{2}\right)$ there is a phenotype $x_{0}$ with maximal carrying capacity. We assume that this optimal phenotype varies linearly with one spatial dimension, $x_{0}\left(z_{1}\right)=\alpha z_{1}+x_{0}(0)$, where $\alpha$ is the slope of the gradient, but is independent of the other spatial dimension $z_{2}$ (Figure 1). Such a resource gradient in one spatial dimension could, for example, represent an altitudinal temperature or humidity gradient along a mountain side that induces a change in the optimal phenotype with altitude. As in the nonspatial model described above, the carrying capacity $K$ takes the normal form,

$$
K=K_{0} \exp \left(-\frac{1}{2}\left[x-x_{0}\left(z_{1}\right)\right]^{2} / \sigma_{K x}^{2}\right),
$$

and is thus a function of both phenotype $x$ and spatial location $z_{1}$ (Figure 1).

As in the well-mixed case, we further assume that competition may be frequency dependent (i.e., the strength of competition between two individuals may depend on their phenotypic distance), so that competition is strongest between individuals with similar phenotypes. In addition, we assume that the strength of competition decreases with spatial distance between individuals. Thus, in our individual-based models the effective population size that determines the death rate of a given individual through competition (Box 1) depends on both the absolute number of other individuals in the neighborhood and on their phenotypes. Specifically, the relative strength $a$ of competition between two individuals with phenotypes $x$ and $x^{\prime}$ and with spatial distance $\Delta z$ between them is given by a product of two normal functions,

$$
a=\frac{1}{2 \pi \sigma_{a z}^{2}} \exp \left(-\frac{1}{2}\left(x-x^{\prime}\right)^{2} / \sigma_{a x}^{2}\right) \exp \left(-\frac{1}{2} \Delta z^{2} / \sigma_{a z}^{2}\right) .
$$


The parameters $\sigma_{a x}$ and $\sigma_{a z}$ thus determine how fast the strength of competition between individuals decreases, respectively, with their phenotypic and spatial distance. We here envisage a situation in which individuals in a given phenotypically monomorphic, but spatially distributed population experience the same total amount of competition for different values of the spatial widths $\sigma_{a z}$ of the interaction kernel. Thus, if $\sigma_{a z}$ is small, spatially very close individuals have a relatively large impact, whereas if $\sigma_{a z}$ is large, the same spatially close individuals have less of an impact and spatially distant individuals become more important in such a way that the total competitive impact is the same. This assumption assures that in the absence of an environmental gradient, equilibrium population sizes of monomorphic populations are independent of $\sigma_{a z}$. This, in turn, corresponds to the biologically reasonable assumption that the equilibrium population size supported by a given spatial distribution of resources is independent of the value of $\sigma_{a z}$, which thus only measures the relative impact of spatially near and far individuals, respectively. This is why the normalizing constant $1 /\left(2 \pi \sigma_{a z}^{2}\right)$ is incorporated into the competition kernel $a$ given by Equation (2b).

Finally, to describe movement in the spatially structured model we assume that individuals can move through the spatial arena over distances and at rates independent of their phenotypes (Box 1). Given that ecological interactions between individuals are localized, populations can become spatially structured if movement occurs rarely or only covers short distances, whereas frequent movement over long distances results in well-mixed and hence spatially unstructured populations. Based on these ecological determinants, the evolutionary dynamics of the quantitative trait $x$ can be investigated. We first do this in asexual populations by allowing for small mutations during birth events (Box 1).

As is explained in Box 1, a total of 11 parameters are needed to describe the spatially structured asexual populations. However, this complexity can be reduced considerably by considering the relevant limiting cases, and by appropriately rescaling units for phenotype, spatial distance, and time. In the salient limit of large spatial areas, and hence large $L$, of large local population sizes, and hence large $K_{0}$, and of small mutations, and hence small values of $u_{\mathrm{as}} \sigma_{\mathrm{as}}^{2}$ (Box 1), we are left with seven relevant parameters. Then, by using $\sigma_{a x}$ as the unit of phenotype and $\sigma_{a z}$ as the unit for space in Equations (2a) and (2b), we are left with the two dimensionless parameters $\alpha \sigma_{a z} / \sigma_{K x}$ and $\sigma_{a x} / \sigma_{K x}$ as determinants of these two equations, respectively. For the remaining parameters $m, \beta$, and $\sigma_{m} / \sigma_{a z}$ (with the latter arising from taking $\sigma_{a z}$ as the unit of spatial distance), note that by taking the birth rate $\beta$ as the unit of time, we are left with a single rate parameter $m / \beta$. In the limit of small movement distances, the compound parameter $(m / \beta)\left(\sigma_{m} / \sigma_{a z}\right)^{2}=$ $\left(\widetilde{\sigma}_{m} / \sigma_{a z}\right)^{2}$ describes spatial movement (where $\widetilde{\sigma}_{m}=\sigma_{m} \sqrt{m / \beta}$ is the expected movement distance during the average lifespan of an individual; see Box 1). This can be seen by considering the deterministic approximation to the individual-based model that results from consideration of the limit of infinite local population size (Box 2). Taking the limit of small movement distances in this deterministic approximation results in a dynamical system in which movement is described solely by the parameter $(m / \beta)\left(\sigma_{m} / \sigma_{a z}\right)^{2}$ [see the movement term in Equation (a) and its expansion given by Equation (b) in Box 2]. In sum, the three essential parameters for our spatially structured populations are the scaled slope of the environmental gradient $\alpha \sigma_{a z} / \sigma_{K x}$, the scaled width of the competition kernel $\sigma_{a x} / \sigma_{K x}$, and the scaled movement distance $\widetilde{\sigma}_{m} / \sigma_{a z}$. In the following, we refer to the latter simply as "mobility".

To aid with the biological interpretation of these dimensionless parameters, we note the following. If the scaled slope of the environmental gradient $\alpha \sigma_{a z} / \sigma_{K x}$ equals 1 , then movement of a capacity maximizing phenotype by $\sigma_{a z}$ in the $z_{1}$ direction reduces its carrying capacity by $1 / e$. If the scaled width of the competition kernel $\sigma_{a x} / \sigma_{K x}$ equals 1 , then the phenotypic distance that reduces the strength of competition by a given amount is the same as the phenotypic distance from the capacity-maximizing phenotype that reduces the carrying capacity by the same amount. Fi- 
Box 1 Individual-based and spatially explicit speciation models

Here we briefly detail the setup of our individual-based stochastic models for spatially structured populations.

- Events. At each computational step, individuals are assigned birth rates $\beta_{i}$, death rates $\delta_{i}$, and movement rates $m_{i}, i=1, \ldots, N$, where $N$ is the current population size; these rates are updated after each event. Based on the total rates $\beta_{\text {tot }}=\sum_{i=1}^{N} \beta_{i}, \delta_{\text {tot }}=\sum_{i=1}^{N} \delta_{i}$, $m_{\mathrm{tot}}=\sum_{i=1}^{N} m_{i}$, and $e_{\mathrm{tot}}=\beta_{\mathrm{tot}}+\delta_{\mathrm{tot}}+m_{\mathrm{tot}}$, the time that elapses until the next event is drawn from an exponential probability distribution with mean $1 / e_{\text {tot }}$, while the type of that event is chosen according to the probabilities $\beta_{\text {tot }} / e_{\text {tot }}, \delta_{\text {tot }} / e_{\text {tot }}$, and $m_{\text {tot }} / e_{\text {tot }}$. The affected individual $i$ is chosen with probability $\beta_{i} / \beta_{\mathrm{tot}}, \delta_{i} / \delta_{\mathrm{tot}}$, or $m_{i} / m_{\mathrm{tot}}$, and the chosen individual either gives birth to one offspring, dies, or performs a spatial movement, depending on the type of event that is occurring.

- Traits and genotypes. In the asexual models, ecological phenotypes $0 \leq x \leq 1$ vary continuously. In the sexual models, phenotypes are determined by up to three sets of diallelic diploid loci with additive effects and free recombination (Chapter 5). The first set of $l_{1}$ loci determines the ecological trait $x$. The second set of $l_{2}$ loci determines a mating trait $y$ that varies between -1 and +1 and determines mating probabilities, which are based either on the ecological trait or on a neutral marker trait (see Birth below). In the latter case, the marker trait also varies between 0 and 1 and is encoded by the third set of $l_{3}$ loci.

- Spatial gradient. Individuals have a spatial location $\left(z_{1}, z_{2}\right)$, with $0 \leq z_{1}, z_{2} \leq L$. We denote by $\phi_{\sigma}(v)=\exp \left(-\frac{1}{2} v^{2} / \sigma^{2}\right)$ and $\widetilde{\phi}_{\sigma}(v)=\phi_{\sigma}(v) / \sqrt{2 \pi} \sigma$, respectively, a normal function and the corresponding normal probability density with mean 0 and variance $\sigma^{2}$. The carrying capacity for the ecological phenotype $x$ at spatial location $\left(z_{1}, z_{2}\right)$ is then given by $K=$ $K_{0} \phi_{\sigma_{K x}}\left(x-x_{0}\left(z_{1}\right)\right)$, where $x_{0}\left(z_{1}\right)=\alpha\left(z_{1}-L / 2\right)+L / 2$ is the phenotype that maximizes $K$ at location $z_{1}$, and $0 \leq \alpha \leq 1$ is the slope of the environmental gradient (Figure 1); $x_{0}$ thus varies over space in the range $[(1-\alpha) L / 2,(1+\alpha) L / 2]$.

- Movement. Individuals move at a fixed rate $m_{i}=m$ and undergo displacements $\Delta z$ in the $z_{1}$ and $z_{2}$ directions independently drawn from $\widetilde{\phi}_{\sigma_{m}}(\Delta z)$, resulting in an average movement distance $\sigma_{m}$. Boundaries are reflective in the $z_{1}$ direction and periodic in the ecologically neutral $z_{2}$ direction. Note that at demographic equilibrium, the expected movement distance during the average lifespan of an individual is $\widetilde{\sigma}_{m}=\sigma_{m} \sqrt{m / \beta}$, where $\beta$ is the birth rate. The reason for the appearance of $\beta$ is that at statistical equilibrium the average lifespan of an individual equals the inverse of the average per capita birth rate, and the model assumptions are such that the life times of the individuals vary with the local circumstances, while the per capita birth rate $\beta$ is constant.

- Death. The effective population size experienced by an individual $i$ with phenotype $x$ at location $\left(z_{1}, z_{2}\right)$ is a weighted sum, $\widetilde{N}=\sum \phi_{\sigma_{a x}}(\Delta x) \frac{\phi_{\sigma_{a z}}(\Delta z)}{2 \pi \sigma_{a z}^{2}}$, where the sum extends over all pairs $(\Delta x, \Delta z)$ of phenotypic and spatial distances between the focal and other individuals. The resultant logistic death rate of individual $i$ is $\delta_{i}=\widetilde{N} / K$.

continued 
Box 1 continued

- Birth. In asexual populations, individuals reproduce at a fixed rate $\beta_{i}=\beta$. Offspring express the parental phenotype unless a mutation occurs with probability $u_{\text {as }}$, in which case their phenotype $x^{\prime}$ is chosen according to $\widetilde{\phi}_{\sigma_{\text {as }}}\left(x^{\prime}-x\right)$. For sexual populations, an individual $i$ slated for reproduction chooses a partner $j$ proportional to phenotype-based mating propensities $p_{i j}$, depending on its mating character and the partner's phenotypic distance in either ecological or marker character as follows (see Figure 5.2 in Dieckmann et al. 2004). If mating propensities depend on the ecological trait, then, for an individual with mating trait $y>0$, mating propensities fall off with a difference $\Delta x$ in the ecological trait of potential mating partners according to $\phi_{\sigma_{+}}(\Delta x)$, where the width of the "mating kernel", $\sigma_{+}$, is given by $\sigma_{+}=\varepsilon / y^{2}$, where $\varepsilon$ is a parameter that indicates how fast assortativeness increases with increasing $y$. Thus, individuals with $y>0$ mate assortatively, with the degree of assortativeness increasing as $y$ approaches the value of 1 . Individuals with mating trait $y=0$ choose partners randomly with respect to phenotype. For individuals with mating trait $y<0$, mating propensities increase with increasing phenotypic distance $\Delta x$ of potential mating partners according to the function $1-\phi_{\sigma_{-}}(\Delta x)$, where the degree of disassortativeness $\sigma_{-}$is determined by the mating trait $y$ as $\sigma_{-}=y^{2}$. The mating propensities of an individual are normalized across all potential partner phenotypes. If mating propensities depend on the marker trait, the distance $\Delta x$ in the ecological trait is replaced by the distance in the marker trait in the formulas above.

In our spatial models, the location-based component $q_{i j}$ of mating propensities decreases according to $\phi_{\sigma_{p}}(\Delta z) /\left(2 \pi \sigma_{p}^{2}\right)$ with the spatial distance $\Delta z$ between potential partners. This induces a cost to the preference for locally rare phenotypes, $\beta_{i}=\beta N_{p} /\left(c+N_{p}\right)$, where $N_{p}=\sum_{j=1, j \neq i}^{N} p_{i j} q_{i j}$ is the number of suitable mating partners locally available to individual $i$, and $c$ determines the cost's strength. Notice that assortativeness often evolves despite this cost. For sexual populations, only females are modeled. In effect, our models therefore describe hermaphroditic organisms. However, the models also apply to populations with separate sexes if males are assumed to have the same density and frequency distributions as females. After recombination, the offspring genotype is subjected to allelic mutations according to a reversal probability $u_{\mathrm{s}}$ per allele. Offspring undergo an initial movement event from the location of their parent.

- Parameters and initial conditions. Unless otherwise stated: $l=10, L=1, K_{0}=500$, $\sigma_{K x}=0.3, \alpha=0.95, \sigma_{a x}=0.9, \sigma_{a z}=0.19, \beta=1, u_{\mathrm{as}}=0.005, \sigma_{\mathrm{as}}=0.05, \sigma_{p}=0.2$, $c=10, u_{\mathrm{s}}=0.001, m=5, \sigma_{m}=0.12$ (i.e., $\tilde{\sigma}_{m} \approx 0.27$ ), and $\varepsilon=0.05$. In the limit of large $L$ and $K_{0}$ and small $u_{\text {as }} \sigma_{\text {as }}^{2}$, the asexual model has no more than three essential dimensionless parameters (see main text): $\sigma_{a x} / \sigma_{K x}, \widetilde{\sigma}_{m} / \sigma_{a z}$, and $\alpha \sigma_{a z} / \sigma_{K x}$.

For the simulations reported here, we used the following initial conditions. In the asexual models, $K_{0}$ individuals were distributed randomly over space and had the phenotype that was optimal in the center of the environmental gradient. In the sexual models, $K_{0}$ individuals were distributed randomly over space, with genotypes assigned randomly assuming allele frequencies of $1 / 2$ at all loci. 


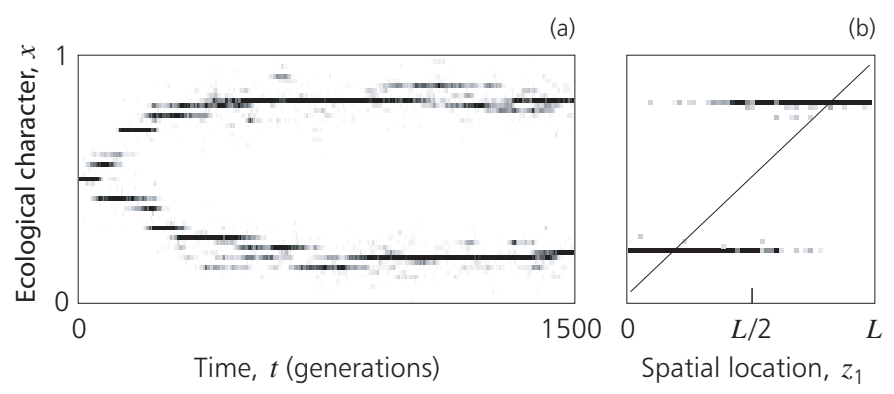

Figure 2 Evolutionary dynamics of adaptive divergence in asexual populations. (a) The distribution of phenotypes as a function of time (using the same shading scheme as in Figure 1). (b) The frequency distribution of phenotypes as a function of spatial location $z_{1}$ at the end of the time series shown in (a). The diagonal line indicates the environmental gradient (see Figure 1). Parameters as given in Box 1.

nally, if the mobility $\widetilde{\sigma}_{m} / \sigma_{a z}$ equals 1 , the expected movement distance during an average lifespan equals $\sigma_{a z}$, the width of the spatial interaction kernel.

Critical aspects of spatial structure are determined by the steepness of the environmental gradient and the mobility. If the gradient is very shallow, the environment becomes essentially spatially homogeneous. If mobility is large, the population becomes well-mixed and hence spatially unstructured. In either of these cases the system behavior approaches that of the nonspatial model: evolutionary branching occurs if $\sigma_{a x}<\sigma_{K x}$, with the individuals belonging to the two different phenotypic clusters scattered throughout the spatial arena.

The system's behavior changes rather drastically if both the spatial resource gradient is steep enough and the mobility is low enough. In this case, if evolutionary branching occurs, it is accompanied by spatial segregation of the diverging phenotypic clusters (Figure 2). Thus, when frequency-dependent interactions occur under conditions of ecological contact and cause spatial evolutionary branching, the environmental gradient serves to organize the new lineages that emerge from this intrinsically sympatric process into geographic abutment (Figure 2). If the process that generates the pattern were not taken into account, the resultant pattern of species abundances would suggest allopatric or parapatric divergence merely driven by local adaptation. But this conclusion would be mistaken: in the absence of frequency-dependent selection, a gradual change in environmental conditions simply results in a gradual change in phenotypic composition (provided, of course, that a sufficiently fine-grained set of phenotypes can be coded for genetically). Therefore, frequency-dependent selection is essential for the emergence of stepped phenotypic clines along the linear environmental gradients in our models.

A second and perhaps more important effect of spatial structure is that, for significantly sloped environmental gradients and low mobility, evolutionary branching occurs over a much wider range of parameters than in the nonspatial models (i.e., for values of $\sigma_{a x}$ that are much larger than $\sigma_{K x}$ ). The degree to which spatial structure facilitates branching and the abrupt onset of this facilitation as a function of mobility (illustrated in Figure 3a) are surprising. If mobility exceeds a certain threshold value, parameter requirements for branching in the spatial and nonspatial models are almost exactly the same. However, as mobility decreases below this threshold, parameter requirements in the spatial model become suddenly and drastically less restrictive than in the nonspatial model. In fact, if mobility is small enough, evolutionary branching occurs even for effectively infinite $\sigma_{a x}$ (i.e., even if there is no intrinsic frequency dependence in the competitive interactions). This is illustrated in Figure 3b, for which the scaled phenotypic width of the competition function is chosen to be very large. Nevertheless, evolutionary branching occurs once mobility falls below a critical level. Interestingly, this critical level depends on the slope of the environmental gradient, and is highest for environmental gradients of intermediate steepness. 
Box 2 Deterministic approximation to the individual-based models

Here we assume that population sizes are locally infinite, both with regard to spatial location and to location in phenotype space. Since we assume a one-dimensional environmental gradient, without differentiation in carrying capacities along the other spatial dimension, the spatially structured population is described by a function $n(x, z, t)$, which denotes the density of phenotype $x$ at spatial location $z$ along the gradient and at time $t$. If, as in $\operatorname{Box} 1, \phi_{\sigma}(x)=\exp \left(-\frac{1}{2} x^{2} / \sigma^{2}\right)$ and $\widetilde{\phi}_{\sigma}(x)=\phi_{\sigma}(x) /(\sqrt{2 \pi} \sigma)$ denote, respectively, a normal function and the corresponding normal density with mean 0 and variance $\sigma^{2}$, the temporal dynamics of the distribution $n(x, z, t)$ can be described by the partial differential equation

$$
\begin{aligned}
\frac{\partial}{\partial t} n(x, z, t)= & \left(1-u_{\mathrm{as}}\right) \beta \int \widetilde{\phi}_{\sigma_{m}}\left(z^{\prime}-z\right) n\left(x, z^{\prime}, t\right) \mathrm{d} z^{\prime} \\
& +u_{\mathrm{as}} \beta \iint \widetilde{\phi}_{\sigma_{\mathrm{as}}}\left(x^{\prime}-x\right) \widetilde{\phi}_{\sigma_{m}}\left(z^{\prime}-z\right) n\left(x^{\prime}, z^{\prime}, t\right) \mathrm{d} x^{\prime} \mathrm{d} z^{\prime} \\
& -n(x, z, t) \frac{\tilde{n}}{K(x, z)} \\
& -m n(x, z, t)+m \int \widetilde{\phi}_{\sigma_{m}}\left(z^{\prime}-z\right) n\left(x, z^{\prime}, t\right) \mathrm{d} z^{\prime} .
\end{aligned}
$$

Here $K(x, z)$ is the carrying capacity of phenotype $x$ at spatial location $z$ (other parameters are as in Box 1). The first term on the right-hand side is the contribution to change in $n(x, z, t)$ from birth events without mutation at location $\left(x, z^{\prime}\right)$, that is, from parents $x$ at spatial location $z^{\prime}$ whose offspring move to $z$. The second term represents the contribution of births to the population density at $(x, z)$ that come from all locations $\left(x^{\prime}, z^{\prime}\right)$ through a mutation of size $x-x^{\prime}$ along the phenotypic axis and a spatial movement of distance $z-z^{\prime}$ along the spatial axis. The third term represents the rate of death caused by competition from individuals at all locations $\left(x^{\prime}, z^{\prime}\right)$. Similar to Box 1 , the effective density experienced by individuals at location $(x, z)$ is given by $\tilde{n}=\iint \phi_{\sigma_{a x}}\left(x^{\prime}-\right.$ $x) \widetilde{\phi}_{\sigma_{a z}}\left(z^{\prime}-z\right) n\left(x^{\prime}, z^{\prime}, t\right) \mathrm{d} x^{\prime} \mathrm{d} z^{\prime}$, so that the per capita death rate is $\tilde{n} / K(x, z)$. The second-to-last term on the right-hand side of Equation (a) describes movement away from spatial location $z$, and the last term describes the effect of movement of phenotype $x$ from all other spatial locations $z^{\prime}$ to spatial location $z$.

System (a) can be simulated numerically and, for suitable parameter combinations, equilibrium distributions $\hat{n}(x, z)$ are obtained that reflect the formation of spatially segregated phenotypic clusters, and thus represent spatial evolutionary branching. However, both the phenotypic and the spatial segregation obtained in the deterministic system are often less sharp than those seen in the individual-based models. In particular, close to the boundary in parameter space that delineates the region in which branching occurs in the individual-based models (see Figure 3), it can happen that branching only occurs in the individual-based model, but not in the deterministic system. This is illustrated in panels (a) and (b) below, for which the same parameter values are used as in Figure 3a, with $\sigma_{m}=0.184$ and $\sigma_{a x}=1$. With these parameters, the deterministic system (left panel) does not branch, in contrast to the individual-based model (right panel). This illustrates that the assumption of locally infinite population sizes made to derive the deterministic approximation is problematic not only for reasons of biological realism, but also because important features of the original model can be lost.

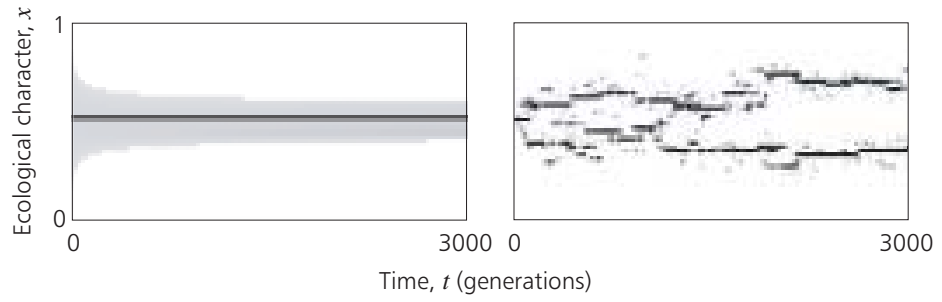




\section{Box 2 continued}

Equation (a) can be simplified further using various approximations. For example, because the distributions $n(x, z, t)$ are continuous, all phenotypes are present at all locations (albeit possibly with very small densities). Therefore, mutations are of lesser importance in the deterministic approximation, and we can simplify Equation (a) by setting $u_{\text {as }}=0$. Furthermore, for small movement kernels $\sigma_{m}$, we can expand the last term on the right-hand side of Equation (a), which describes movement to location $z$, to obtain the second-order approximation

$$
\int \widetilde{\phi}_{\sigma_{m}}\left(z^{\prime}-z\right) n\left(x, z^{\prime}, t\right) \mathrm{d} z^{\prime} \approx n(x, z, t)+\frac{1}{2} \sigma_{m}^{2} n_{z z}(x, z, t),
$$

where $n_{z z}(x, z, t)$ denotes the second partial derivative of $n(x, z, t)$ with respect to $z$. It turns out that one cannot take advantage of a similar expansion for small $\sigma_{a x}$ and $\sigma_{a z}$, since this renders Equation (a) dynamically unstable. This problem is avoided when $\sigma_{a x}$ and $\sigma_{a z}$ are not assumed merely to be small, but are assumed to vanish altogether (the phenotypic and spatial components of the interaction kernel are then given by Dirac delta functions). Box 3 presents results for yet another limiting case. Numerical analysis of such simplified systems is generally much more convenient than using individual-based stochastic simulations and may thus enable a quicker exploration of the underlying parameter space. In addition, such systems are also more likely to yield analytical insights.

The mechanisms that generate these effects can be illustrated as follows. An environmental gradient initially induces gradual spatial differentiation caused by local adaptation along the gradient. Thus, local adaptation implies a correlation between spatial location and phenotype. When, as assumed here, significant competition occurs only between individuals that are spatially sufficiently close, this correlation decreases the strength of competition between phenotypically distant individuals, and hence increases the degree of frequency dependence in the system. Such gradient-induced frequency dependence can lead to evolutionary branching, even if the phenotypic width of the competition function is very large. The effect tends to be weaker if local adaptation is very incomplete because of gene flow along shallow gradients, or if dissimilar phenotypes are spatially close because of local adaptation along a very steep environmental gradient. Therefore, facilitation of evolutionary branching through gradient-induced frequency dependence is highest for intermediate environmental gradients, as illustrated in Figure 3b. In this figure, frequency dependence results entirely from localized interactions between spatially differentiated individuals, and no evolutionary branching at all is expected in the nonspatial model.

The individual-based asexual models are characterized by three essential parameters, so we can use the information provided by Figures $3 \mathrm{a}$ and $3 \mathrm{~b}$ to represent system behavior schematically in a three-dimensional plot. This characterization of the branching behavior of the system is shown in Figure 3c, which has as axes the three dimensionless parameters $\alpha \sigma_{a z} / \sigma_{K x}$ (scaled slope of the environmental gradient), $\sigma_{a x} / \sigma_{K x}$ (scaled width of the competition kernel), and $\widetilde{\sigma}_{m} / \sigma_{a z}$ (scaled movement distance, that is, mobility). Figures $3 \mathrm{a}$ and $3 \mathrm{~b}$ are indicated in Figure $3 \mathrm{c}$ as planar cross sections of the three-dimensional solid that represents the parameter combinations that lead to spatial evolutionary branching. Figure $3 c$ was extrapolated from Figures $3 a$ and $3 b$ using additional simulations to determine the height and the position of the central ridge. Figure 3 again makes it clear that evolutionary branching is facilitated greatly in spatially structured populations. The central ridge in Figure 3c illustrates that facilitation of evolutionary branching is generally greatest for environmental gradients of intermediate slope. More precisely, evolutionary branching is most likely for parameter combinations for which the scaled slope of the gradient $\alpha \sigma_{a z} / \sigma_{K x}$ is approximately equal to 1 . This observation may serve as a starting point for empirical tests of our models, for we thus expect evolutionary diversification to be most likely for organisms and environments for which $\alpha=\sigma_{K x} / \sigma_{a z}$. In as much as the quantities $\alpha$ (the steepness of an environmental gra- 

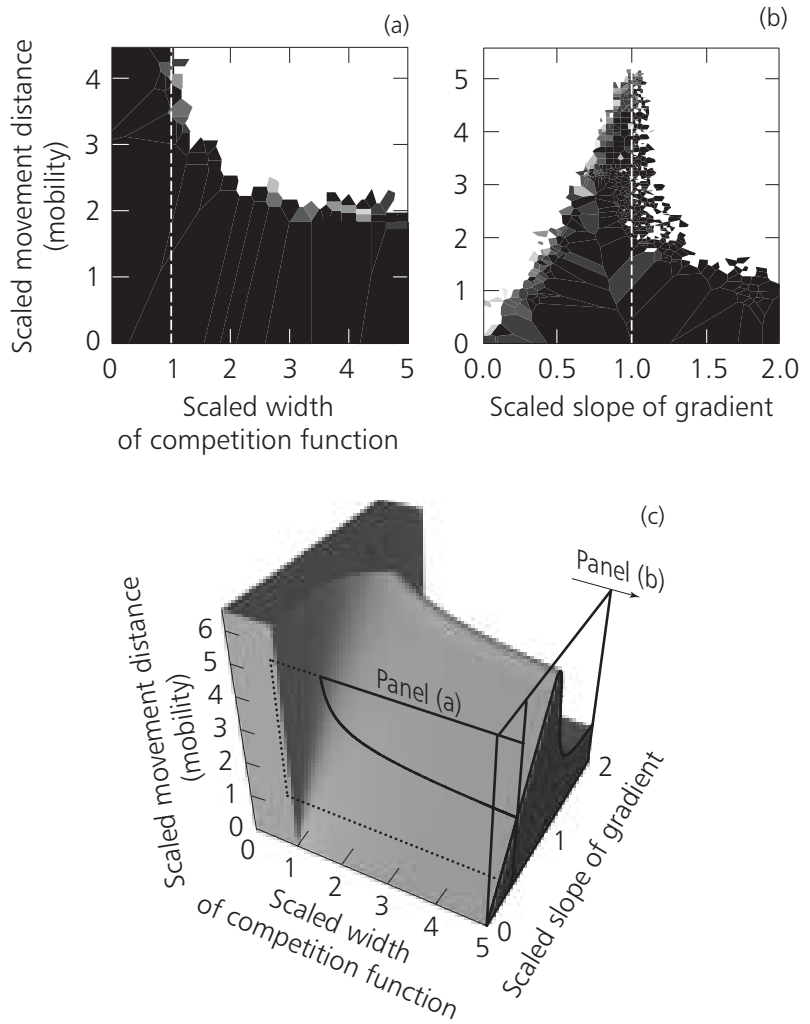

Figure 3 Requirements for evolutionary branching in asexual populations. The axes correspond to the model's three dimensionless parameters (see Box 1): scaled width of competition function $\sigma_{a x} / \sigma_{K x}$, scaled movement distance (mobility) $\sqrt{m / \beta} \sigma_{m} / \sigma_{a x}$, and scaled slope of gradient $\alpha \sigma_{a x} / \sigma_{K x}$. (a) and (b) show a subdivision of parameter space into polygons (Voronoi tessellation based on simulation data), shaded according to the recorded time to evolutionary branching: black corresponds to branching within the first 500 generations, white corresponds to no branching after 5000 generations, and shades of gray correspond to branching between generations 500 and 5000 (including multiple branching, which occurs for very low movement distances). (a) Effect of direct frequency dependence. Variation of time until branching with scaled width of competition function and mobility (scaled movement distance) for asexual populations (at $\left.\alpha \sigma_{a x} / \sigma_{K x}=0.425\right)$. In nonspatial models, only parameter combinations to the left of the dashed line are expected to induce branching. (b) Effect of gradient-induced frequency dependence. Variation of time until branching with scaled slope of gradient and mobility for asexual populations with $\sigma_{a x} \gg \sigma_{K x}$. In nonspatial models no branching is expected at all. (c) Complete characterization of asexual model. Evolutionary branching occurs for parameters within the shaded block. The positions of the slices shown in panels (a) and (b) are indicated.

dient), $\sigma_{K x}$ (the phenotypic width of the carrying capacity function along the gradient), and $\sigma_{a z}$ (the width of the spatial interaction kernel) are measurable in natural populations, this observation could serve as a basis for comparative studies of diversity in different taxa.

An obvious limitation of the analysis presented in Figure 3 is that this analysis is based on numerical simulations. It would clearly be very useful to have an analytical theory for the evolutionary dynamics of our spatially structured populations, for such a theory might, for example, allow us to derive analytical criteria for spatial evolutionary branching as a function of parameter values. In fact, by assuming locally infinite population sizes, for which "local" must be understood both spatially and phenotypically, it is possible to derive a deterministic approximation of the individual-based models (Box 2). Although the resultant partial differential equation is, again, amenable to numerical investigation only, it would, in principle, allow for a more tractable investigation. However, results from the deterministic approximation differ considerably from those obtained with the individual-based models. In particular, the sharp spatial segregation between 
Box 3 Evolutionary branching in a reaction-diffusion system

Ferenc Mizera and Géza Meszéna

Here we consider the deterministic model for evolution along a spatial gradient given by Mizera and Meszéna (2003). The gradient is similar to those used in the individual-based models of Section 3 , but incorporates the additional realistic assumption that the maximal carrying capacity $K_{0}$ is a function with an intermediate maximum along the gradient. Thus, not only does the phenotype that maximizes the carrying capacity change along the gradient, but the phenotypically maximal carrying capacity itself has a maximum at the center of the spatial axis that defines the gradient. We assume that this unimodality in the phenotypically maximal carrying capacity is given by

$$
K_{0}(z)=K_{1} \exp \left(-\frac{1}{2} z^{2} / \sigma_{K z}^{2}\right)
$$

where $\sigma_{K z}$ describes how fast the maximal carrying capacity $K_{0}(z)$ declines from the optimum $K_{1}$ with spatial distance from the center of the gradient at $z=0$.

In addition, we assume that the slope $\alpha$ of the environmental gradient equals 1 , so that we can identify phenotype with spatial location. Thus, the carrying capacity of phenotype $x$ at spatial location $z$ is given by

$$
K(x, z)=K_{0}(z) \exp \left(-\frac{1}{2}(x-z)^{2} / \sigma_{K x}^{2}\right) .
$$

We also assume that there is no intrinsic frequency dependence in the competitive interactions (i.e., we consider the limit $\sigma_{a x} \rightarrow \infty$ ), and that interactions only occur between individuals inhabiting the same spatial location $z$, but not between individuals from different spatial locations (i.e., we consider the limit $\sigma_{a z} \rightarrow 0$ for the width of the spatial interaction kernel). Finally, birth, death, and movement are calculated as described in Box 1, except we assume that the offspring have the same spatial position as their parent.

The adaptive dynamics of the trait $x$ can be studied using invasion analysis (Chapter 4 in Dieckmann et al. 2004) based on a deterministic reaction-diffusion equation. If $n\left(x^{\prime}, z, t\right)$ denotes the density of a mutant phenotype $x^{\prime}$ at spatial location $z$ and at time $t$, the dynamics of the distribution $n\left(x^{\prime}, z, t\right)$ is given by

$$
\frac{\partial n\left(x^{\prime}, z, t\right)}{\partial t}=r\left[1-\hat{n}(x, z) / K\left(x^{\prime}, z\right)\right] n\left(x^{\prime}, z, t\right)+D \frac{\partial^{2}}{\partial z^{2}} n\left(x^{\prime}, z, t\right),
$$

where $\hat{n}(x, z)$ is the equilibrium distribution of the resident phenotype $x$ along the gradient, and $r$ is the birth rate. Spatial movement is described by the diffusion coefficient $D=\frac{1}{2} m \sigma_{m}^{2}$, where $m$ is the rate of spatial movement and $\sigma_{m}$ is the average movement distance.

Using a numerical invasion analysis by determining the growth rate of mutants $x^{\prime}$ that appear in monomorphic populations of residents $x$, it can be shown that $x_{0}=0$ is always a convergence-stable singular strategy. This is simply a consequence of the total resource availability being highest at the center $z=0$ of the spatial axis. In addition, it can be shown that the evolutionarily singular attractor $x_{0}=0$ is evolutionarily stable if the diffusion coefficient $D$ and the environmental tolerance $\sigma_{K x}$ are large. However, when the diffusion coefficient and/or the environmental tolerance become small, the singular strategy becomes evolutionarily unstable, and hence becomes an evolutionary branching point. This is illustrated in panel (a) below, which shows the boundary between evolutionary stability and instability of the singular attractor $x_{0}=0$ in the two-dimensional parameter space given by the environmental tolerance $\sigma_{K x}$ and the diffusion coefficient $D$. (Other parameter values used for the panel below are $\sigma_{K z}=0.2$ and $r=1.0$.)

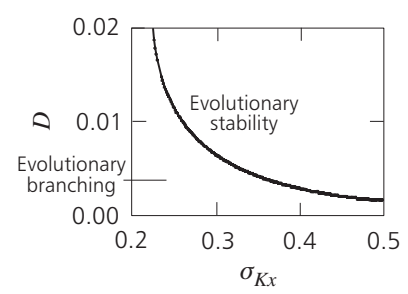




\section{Box 3 continued}

Individual-based simulations of this model confirm that if the singular point is an evolutionary branching point, and if evolutionary dynamics are started from a monomorphic population away from the singular point, the trait $x$ first converges to the singular point, after which it splits into two phenotypic clusters. An example of such evolutionary dynamics is shown in the panels below, in which panel (a) shows the frequency distributions of phenotypes, indicated by shading along the horizontal axis, as a function of time, which runs along the vertical axis. Panel (a) illustrates that after an initial convergence to the singular point $x_{0}=0$, repeated evolutionary branching can occur in this system. Panels (b), (c), and (d) show the density distribution across space of the populations that represent the most abundant phenotype in each of the three branches present at the end of the time series shown in panel (a). (Parameter values for the panels are $D=610^{-5}, \sigma_{K x}=0.3$, $\sigma_{K z}=0.25$, and $r=0.01$.) Note that the emerging lineages exhibit spatial segregation along the environmental gradient. This again illustrates how environmental gradients in resource availability can generate parapatric patterns of species distributions because of intrinsically sympatric ecological processes.
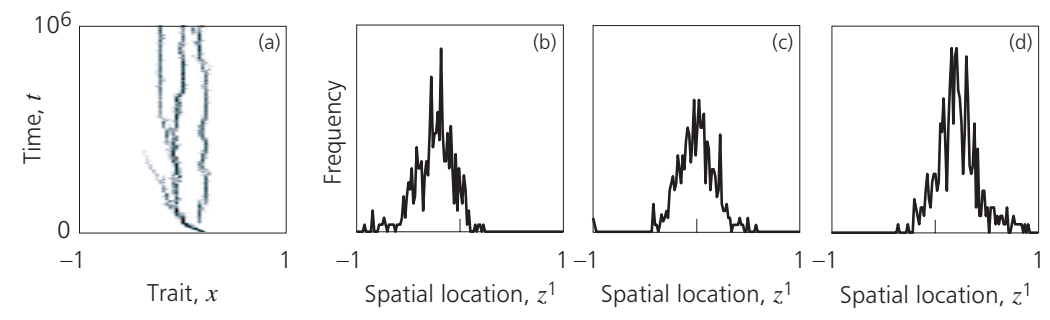

lineages that emerge from evolutionary branching, as shown in Figure 2, are blurred in the deterministic approximations (Box 2). This, in turn, implies that the sharp bifurcation boundaries for evolutionary branching shown in Figure 3 are washed out in the deterministic system, so that the critical threshold in parameter values is less clear. Moreover, even when accounting for this artifact, the deterministic system also predicts the boundary's location inaccurately. These quantitative differences may arise from taking the limit of infinite local population sizes in both spatial and phenotypic dimensions (an assumption that appears to be even more difficult to justify biologically than the often used limit of infinite global population size). As a result, the implications of reproductive (and other) pair correlations, of local density fluctuations, and of demographic stochasticity are ignored, all of which have been shown to affect ecological and evolutionary dynamics critically (Dieckmann et al. 2000).

It appears to be rather difficult to extract analytical results from such deterministic approximations, but they lend themselves to more tractable numerical analysis and, for example, enable quicker searches of parameter space. A useful application of this approach is explained in Box 3, which shows that under certain assumptions spatial evolutionary dynamics can be studied using quite simple deterministic systems. In general, however, the individual-based implementation appears to be inevitable if artifacts caused by biologically unrealistic assumptions are to be avoided. This is particularly true for the investigation of evolutionary dynamics in spatially structured sexual populations with multilocus genetics, which are considered in Section 4.

\section{Extension to Sexual Populations: Parapatric Speciation}

To address the question of parapatric speciation, the individual-based spatially structured models can be extended to describe sexual populations in which the quantitative trait $x$ is determined additively by a number of diploid loci (Box 1). Here the genetic assumptions are the same as 


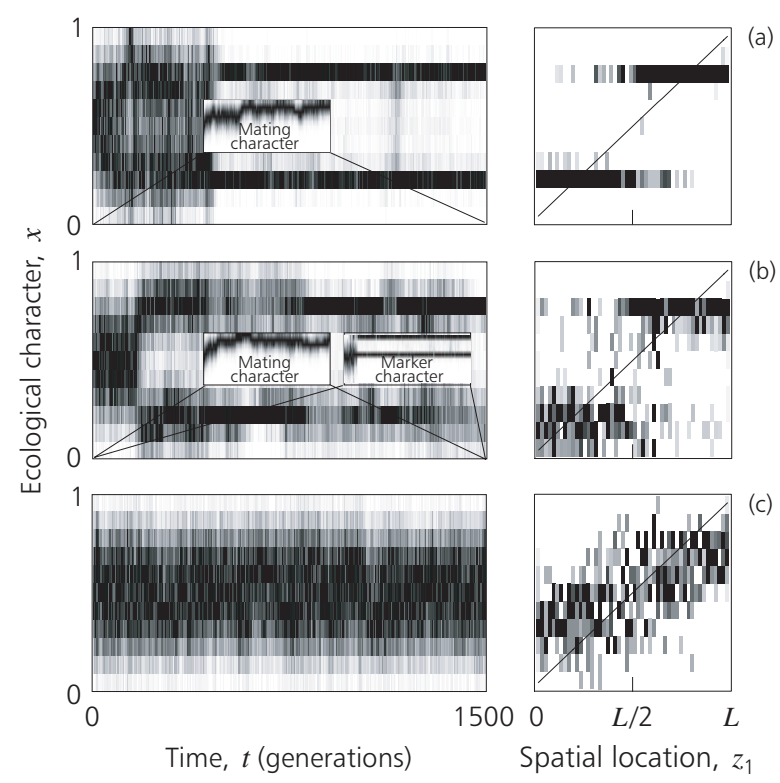

Figure 4 Evolutionary dynamics of adaptive divergence in sexual populations. The left panels show the distribution of phenotypes as a function of time (same shading scheme as in Figure 1) and the right panels show the corresponding frequency distribution of phenotypes as a function of spatial location $z_{1}$. The diagonal lines indicate the environmental gradient (see Figure 1). (a) Evolutionary branching with spatial segregation in a sexual population with the same parameter values as in Figure 2 and with assortative mating based on ecological similarity. The evolution of the degree of assortativeness is shown as an inset in the left panel (intermediate values of the mating trait correspond to random mating, low values to disassortative mating, and high values to assortative mating). Parameters as given in Box 1. (b) Evolutionary branching with spatial segregation in a sexual population with assortative mating based on a marker trait. The evolution of assortativeness and the branching in the marker trait are shown as insets in the left panel (the two marker branches are in linkage disequilibrium with the two branches of the ecological trait). Parameters as given in Box 1, except for $\alpha=0.9, \sigma_{a x}=0.5$, and $\sigma_{m}=0.07$. (c) Evolution of a phenotypic gradient in a sexual population with random mating. Parameters as in (b), except that random mating with respect to phenotype was enforced.

those in Dieckmann and Doebeli (1999) and are described in Box 1. The ecological processes remain the same as before, but instead of reproducing clonally, individuals now depend on having suitable partners available within a given spatial neighborhood. The mating system then influences whether evolutionary branching, and hence parapatric speciation, is possible:

- If mating is random with respect to phenotypes, evolutionary branching no longer occurs, regardless of the relative magnitude of the parameters $\sigma_{a x}$ and $\sigma_{K x}$. Just as in the nonspatial model (Chapter 5 in Dieckmann et al. 2004), random mating brings about recombination between extreme phenotypes, which prevents the evolution of a phenotypic dichotomy.

- It has been shown before that evolutionary branching in well-mixed sexual populations is possible if the evolution of assortative mating is allowed for (Dieckmann and Doebeli 1999). Here we consider the same two general scenarios for assortative mating as described in Chapter 5 in Dieckmann et al. 2004. In the first scenario, assortative mating is based on a similarity in the trait $x$ that determines the ecological interactions; in the second scenario assortative mating is based on an ecologically neutral marker trait (in the latter case, a linkage disequilibrium between the marker trait and the ecological trait must evolve for evolutionary branching to occur). As shown in Chapter 5 in Dieckmann et al. 2004, evolutionary branching in well-mixed sexual populations can occur because of evolution of both types of assortative mating (with the parameter requirements being more restrictive when assortative mating is based on a marker trait). These conclusions essentially carry over to the spatial 
Box 4 Evolution on linear gradients in randomly mating sexual populations

In this box we briefly review the models of Kirkpatrick and Barton (1997) and of Case and Taper (2000) for the evolution of a quantitative trait along a linear environmental gradient. These models show that in a single species with random mating the linear environmental gradient induces a corresponding phenotypic gradient in the quantitative trait, and that interspecific competition can lead to spatial segregation of the competing species along the environmental gradient.

The models are formulated as partial differential equations with diffusive movement of individuals along one spatial dimension $z$. At spatial location $z$ population growth is logistic, and the local population growth rate decreases with increasing distance between the mean phenotype $\bar{u}(z)$ and an optimum phenotype $x_{0}(z)$, which varies linearly with $z$. Thus, populations are only viable at location $z$ if their mean $\bar{u}(z)$ is sufficiently close to the optimum $x_{0}(z)$. Specifically, the local dynamics at location $z$ are of the form

$$
\left(\frac{\mathrm{d} n(z)}{\mathrm{d} t}\right)_{\text {local }}=r n(z)[1-n(z) / K]-\left[\bar{u}(z)-x_{0}(z)\right]^{2} /\left(2 \sigma_{\mathrm{sel}}^{2}\right),
$$

where $n(z)$ is the population density at location $z$, and $\sigma_{\text {sel }}$ measures the strength of stabilizing selection toward $x_{0}$. The first term on the right-hand side of Equation (a) describes logistic growth, and the second term describes how growth rates decrease as the distance between the mean and the optimal phenotype at location $z$ increases. According to the model of Lande (1979b), the local evolution of the mean phenotype is assumed to be given by

$$
\left(\frac{\mathrm{d} \bar{u}(z)}{\mathrm{d} t}\right)_{\text {local }}=G \sigma_{\text {sel }}^{-2}\left[x_{0}(z)-\bar{u}(z)\right],
$$

where $G$ is the product of the heritability with the phenotypic variance of the trait and is assumed to be constant over space and time. The role of $\sigma_{\text {sel }}$ is akin to that of $\sigma_{K x}$ in our individual-based models. Equations (a) and (b) are complemented by diffusive movement along the $z$ axis, characterized as in Box 2 by a diffusion coefficient $D=\frac{1}{2} m \sigma_{m}^{2}$, where $m$ is the rate of spatial movement and $\sigma_{m}$ is the average movement distance. It is assumed that competition only occurs between individuals that exist at the very same spatial location, and that intrinsic frequency dependence in local interactions is absent. This leads to the following system of partial differential equations to describe population growth and evolution along the environmental gradient,

$$
\begin{aligned}
\frac{\partial n}{\partial t} & =D \frac{\partial^{2} n}{\partial^{2} z}+\left(\frac{\mathrm{d} n}{\mathrm{~d} t}\right)_{\text {local }}, \\
\frac{\partial \bar{u}}{\partial t} & =D \frac{\partial^{2} \bar{u}}{\partial^{2} z}+2 D \frac{\partial \ln n}{\partial z} \frac{\partial \bar{u}}{\partial z}+\left(\frac{\mathrm{d} \bar{u}}{\mathrm{~d} t}\right)_{\text {local }} .
\end{aligned}
$$

The first term on the right-hand side of Equation (c) and the first two terms on the right-hand side of Equation (d) represent the effects of dispersal and concomitant gene flow, while the remaining terms represent the local dynamics described in Equations (a) and (b), respectively.

Kirkpatrick and Barton (1997) used this deterministic model to show that if dispersal is large enough, then gene flow swamping the boundary areas of a species range from the center can prevent local adaptation to the optimal phenotype near the boundaries, so that local equilibrium population densities decline to zero toward the species' boundaries. Therefore, gene flow can prevent range expansion. The resultant ecological and evolutionary equilibrium is shown schematically in panel (a) below, which illustrates local adaptation along a linear environmental gradient in the form of a linear phenotypic gradient in the mean trait value. As a result of gene flow from the center of the species range, the phenotypic gradient exhibits increasing distances from the optimal phenotype toward the edges of the spatial area.

continued 
Box 4 continued

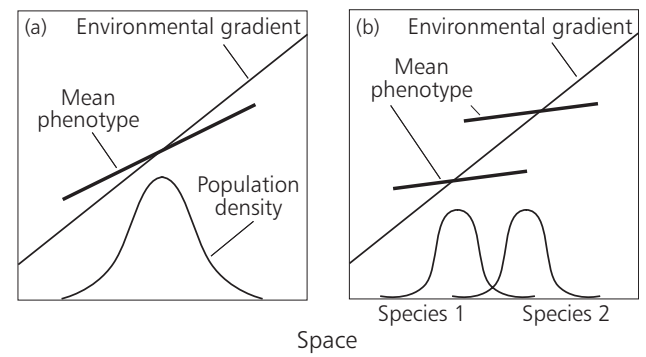

Case and Taper (2000) extended this model to two competing species. One of their main results is that character displacement in the contact zone between the species can lead to even larger degrees of local maladaptation, which thus limits species' ranges even further. This typically results in the two species occupying different spatial areas, and hence leads to spatial segregation along the environmental gradient. A schematic representation of this scenario is shown in panel (b) above, which is the same as panel (a) except that now the spatial arena is occupied by two competing species. Panel (b) illustrates that in this case species ranges are generally smaller because of character displacement as a consequence of interspecific competition in regions of overlap between the two species. Note that, again, the mean phenotype in both species always shows a continuous gradient. The model of Case and Taper (2000) demonstrates that interspecific frequency dependence can induce patterns of spatial segregation between two competing species, a finding in good agreement with the dynamics of our speciation models, in which intraspecific competition is dynamically and gradually transformed into interspecific competition.

model studied in this chapter; the resultant speciation processes are illustrated in Figures 4a and $4 \mathrm{~b}$ for both types of assortative mating.

Geographic differentiation in the presence of a spatial gradient has also been observed by Case and Taper (2000) in a model for competition between two species (Box 4). That model did not, however, address the question of initial diversification in a single population (instead, the existence of two separate species was assumed a priori). Indeed, the type of model used by Case and Taper (2000) serves to illustrate that if random mating prevents diversification of a single species that evolves along a linear gradient, then the evolution of a linear phenotypic gradient that tracks the environmental gradient is expected. Thus, when Kirkpatrick and Barton (1997) earlier on applied the same formalism to a single species to study the problem of species ranges, they found that a linear environmental gradient in the optimal phenotype leads to the evolution of a corresponding phenotypic gradient (Box 4). The same observation can also be made for our model: in the absence of evolutionary branching, a phenotypic gradient evolves (Figure 4c). We can thus conclude that the speciation process described here critically depends on evolutionary branching and on its release through the evolution of assortative mating; both of these processes are triggered by disruptive selection that emerges dynamically from frequency-dependent interactions under conditions of ecological contact. These strictly local interactions can thus ultimately drive the evolutionary diversification of ancestral populations into globally segregated species.

Once the degree of spatial structure caused by low mobility reaches a critical threshold, speciation through spatial evolutionary branching in sexual populations again occurs over a much wider range of parameters than in the corresponding nonspatial sexual populations. This is shown in Figure 5 for the case in which assortative mating is based on similarity in the ecological trait. Also, regarding the effect of the gradient's slope - derived in Section 3 from the numerical analysis of the spatially extended asexual model (Figure 3b) - we find expectations corroborated. As in the asexual case, speciation is especially facilitated for environmental gradients of intermediate slope 

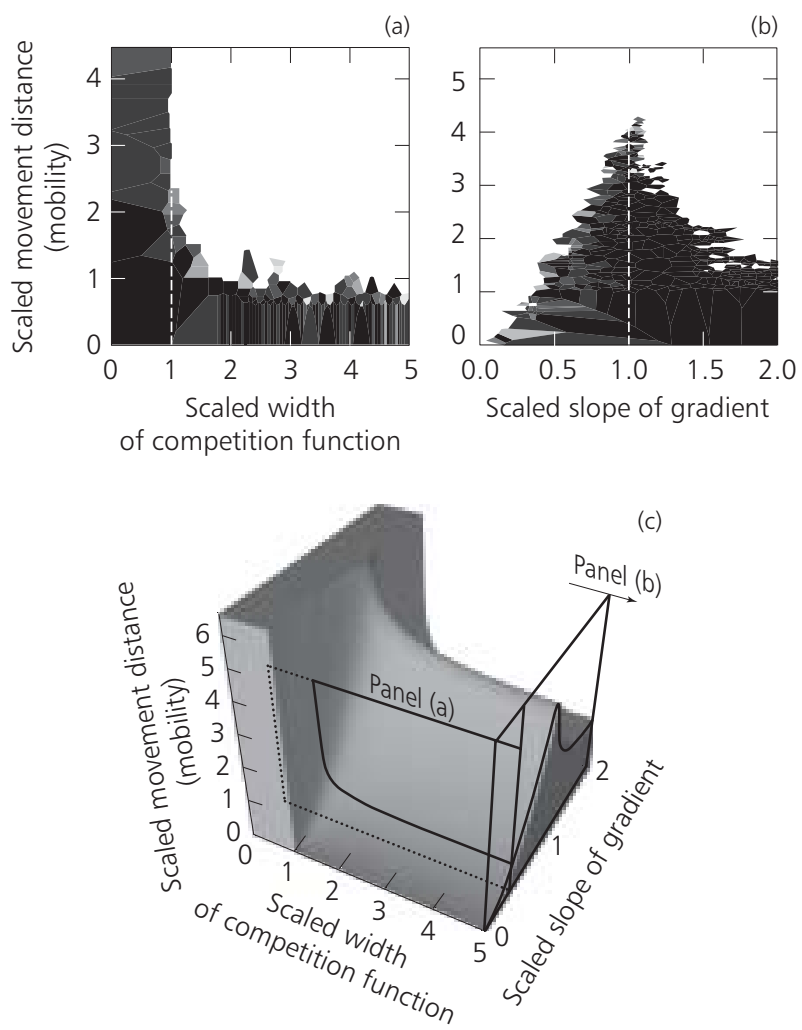

Figure 5 Requirements for evolutionary branching in sexual populations in which assortative mating is based on the ecological trait. The basic setup of this figure is the same as that for Figure 3. The model's three dimensionless parameters (see Box 1) are displayed on all axes. Panels (a) and (b) show a subdivision of parameter space into polygons, shaded according to the recorded time to evolutionary branching: black corresponds to branching within the first 500 generations, white corresponds to no branching after 5000 generations, and shades of gray correspond to branching between generations 500 and 5000. (a) Effect of direct frequency dependence. Variation of time until branching with scaled width of competition function and scaled movement distance (mobility) for asexual populations (at $\alpha \sigma_{a x} / \sigma_{K x}=0.425$ ). In nonspatial models only parameter combinations to the left of the dashed line are expected to induce branching. (b) Effect of gradient-induced frequency dependence. Variation of time until branching with scaled slope of gradient and mobility for asexual populations with $\sigma_{a x} \gg \sigma_{K x}$. In nonspatial models no branching would be expected at all. (c) Behavior of sexual model. Evolutionary branching occurs for parameters within the shaded block. The positions of the slices in panels (a) and (b) are indicated.

(Figures $5 \mathrm{~b}$ and $5 \mathrm{c}$ ). As can be seen by comparing Figures 3 and 5, parameter ranges that allow spatial evolutionary branching in sexual populations are slightly smaller than the corresponding ranges for clonal populations.

Our assumptions that assortative mating originates from mate choice based on phenotypic similarity between partners (either in an ecologically relevant trait or an ecologically neutral marker trait) conform with premating isolation mechanisms investigated in previous analyses of reinforcement (e.g., Liou and Price 1994; Servedio 2000). Other isolation mechanisms, such as mate choice based on absolute preference (Servedio 2000) or on male traits and female preferences (Chapter 5 in Dieckmann et al. 2004), have yet to be considered in the context of evolutionary branching in spatially structured sexual populations. Compared against these more mechanistic models of assortative mating, the approach taken in this chapter must be interpreted as being more phenomenological. This, incidentally, has the advantage of mimicking many different mechanistic modes of assortativeness, which would otherwise need to be studied in different specific models.

We have thus arrived at a description of parapatric speciation as a dynamic consequence of two intertwined processes. First, spatially localized and frequency-dependent interactions along the 
environmental gradient can be the driving force of evolutionary divergence. Second, a dynamics akin to reinforcement causes the evolution of assortative mating. Both processes together allow an ancestral sexual population to become organized into phenotypically distinct descendant species that occupy different, spatially segregated regions along the environmental gradient, and thus result in a stepped phenotypic cline. These results provide an intrinsic ecological explanation for parapatric divergence in geographically continuous populations by linking local processes to the resultant global patterns of species abundance.

\section{A Note on Species-Area Relationships}

The results presented in Sections 3 and 4 show how spatial structure facilitates parapatric speciation. It is therefore tempting to ask whether these findings also have implications for the general problem of understanding species-area relationships. It is an empirically well-documented fact that species diversity tends to increase with the size of the area over which diversity is sampled, which leads to the characteristic species-area relationships often described by power laws (Rosenzweig 1995). This is one of the most ubiquitous patterns found in ecology, and many alternative explanations have been conjectured to date. The suggested mechanisms are likely to complement, rather than exclude, each other, so that observed species-area relationships presumably are the product of the joint action of several disparate mechanisms.

A classic explanation by MacArthur and Wilson (1967) is based on their "equilibrium model of island biogeography", that is, on the assumption that equilibrium population sizes linearly increase with island size, so that the extinction of species occurs more rarely on larger islands. This perspective is purely ecological and makes no reference to the effect of island area on the rate at which species are being formed, rather than being destroyed. By contrast, it has also been suggested that speciation occurs at higher rates in larger areas. For example, Losos and Schluter (2000) recently argued that the greater species richness of Anolis lizards found on larger islands in the Antilles results from higher speciation rates on larger islands, rather than from higher immigration rates from the mainland, or from lower extinction rates. Higher speciation rates in larger areas are, in turn, often attributed to an increased resource and habitat diversity supposedly harbored by larger areas.

Utilizing the spatial speciation model for asexual populations introduced above, here we illustrate that even when there is no difference in resource diversity, larger areas are expected to promote speciation. We suggest that such a relation holds because, according to the speciation mechanism discussed in this chapter, a given characteristic scale of individual mobility enables a higher degree of evolutionary self-structuring along longer gradients, even when these gradients span the same phenotypic range. To test this hypothesis we performed simulations as for Figure 3a, but in a spatial arena scaled down to half the area used for Figure 3a while retaining the gradient's phenotypic span. To achieve this we increased the slope of the environmental gradient in the smaller spatial arena to $\sqrt{2}$ times the slope of the gradient used for Figure 3a. The resultant parameter ranges for evolutionary branching are shown in Figure 6 and can be compared to the results for the larger spatial area in two ways:

- Figure 6 can be compared directly to Figure $3 a$, that is, to results for a larger spatial area that harbors the same resource diversity (i.e., the same range of phenotypes that locally maximize resource utilization).

- Alternatively, the ranges shown in Figure 6 can be compared to those for a larger area with the same slope of the environmental gradient. This implies, however, that the resource optima in the larger area span a larger phenotypic range, so that resource diversity is increased together with the area. In this case, Figure 6 should be compared to a corresponding figure for the larger area that is obtained as a cross-section of Figure $3 \mathrm{c}$, taken parallel to the $x-z$ 


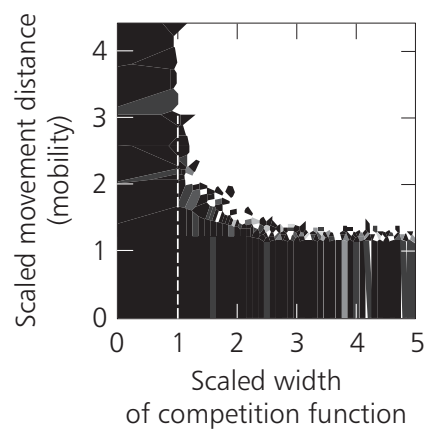

Figure 6 Requirements for evolutionary branching for asexual populations that occupy half the spatial arena used for Figure 3a. As in Figure 3a, the panel shows a subdivision of parameter space into polygons, shaded according to the recorded time to evolutionary branching. The panel shows variation of time until branching with scaled width of competition function and with scaled movement distance (mobility). To ensure the same overall resource diversity as in the larger area used for Figure $3 \mathrm{a}$, the parameter value for the scaled slope of the environmental gradient was set at $\alpha \sigma_{a x} / \sigma_{K x}=\sqrt{2} 0.425$ in the smaller arena. Note that evolutionary branching occurs for a smaller range of parameters than in Figure $3 \mathrm{a}$.

plane at a $y$ value (where $y$ is the gradient slope) that is $\sqrt{2}$ times the $y$ value that corresponds to the cross-section representing Figure $3 a$. Note that a cross-section at $\sqrt{2}$ times the gradient slope used for Figure 3 a would predict larger ranges of parameter values that lead to evolutionary branching than does Figure 3 a.

Whatever the comparison, Figure 6 shows that for low mobility, evolutionary branching still occurs much more readily in the smaller spatial area than in spatially unstructured populations. However, comparison either with Figure 3a directly, or with the cross-section of Figure $3 \mathrm{c}$ at $\sqrt{2}$ times the gradient slope used for Figure 3a, shows that branching occurs for a substantially smaller range of parameters than in a larger spatial area, irrespective of whether the larger area is assumed to have the same resource diversity or the same gradient slope as the smaller area. This means that there are mobilities that allow for greatly facilitated branching in the larger spatial area, but that are too large to have the same effect in the smaller area. Therefore, in our model parapatric speciation rates are higher in larger areas. Our interpretation of this result is that relatively more localized ecological interactions are more conducive to local adaptive processes of diversification. This should not be confused with mechanisms of isolation by distance, in which local processes at spatially very distant locations lead to diversification. In contrast, in our models divergence requires local ecological contact. Sympatric ecological interactions drive local adaptive diversification, and our results suggest that such processes of adaptive speciation are more likely with more localized interactions.

With regard to the Anolis lizards on Caribbean islands, the mechanism described here may be responsible for some aspects of the species-area relationships discussed by Losos and Schluter (2000). Indeed, resource diversity does not appear to be significantly lower on smaller islands in the Antilles (Roughgarden 1995). Yet the large islands of the Greater Antilles typically harbor many species of Anolis lizards, while the smaller islands of the lesser Antilles contain at most two species - although morphological variation within these species can be quite large (Malhotra and Thorpe 1997c; Chapter 16 in Dieckmann et al. 2004). These observations conform with the notion that on the larger islands new species can arise more easily out of existing genetic variation, because evolutionary branching and the concomitant evolution of reproductive isolation is more likely in larger spatial areas. 
Box 5 Fisherian runaway processes in spatially extended populations

Payne and Krakauer (1997) studied models for parapatric speciation that involve divergent Fisherian runaway processes driven by sexual selection in different regions along a spatial gradient. In these models, two alternative marker traits in males correspond to two alternative preference traits in females. In nonspatial models for homogeneous populations, sexual selection is expected to lead to fixation of either one of the two marker-preference pairs, with the actual outcome depending on the initial conditions.

By contrast, a spatial gradient in viability selection that favors one male marker trait to the left of a given spatial location $x_{0}$ and the other male marker trait to the right of $x_{0}$ can lead to spatial pattern formation, with one marker-preference pair evolving to the left of $x_{0}$ and the other evolving to the right of $x_{0}$; this generates a zone of intermediate frequencies with strong linkage disequilibrium between the two spatially segregated species (Lande 1982; Payne and Krakauer 1997). The steeper the environmental gradient, the narrower is this zone, and hence the more likely is complete reproductive isolation and thus speciation.

Payne and Krakauer (1997) also suggested that a spatial gradient in viability selection is not necessary to produce divergent runaway processes in different spatial locations. Instead, they proposed that alternative and spatially segregated marker-preference pairs could also evolve and persist in separate domains for extended periods of time, if dispersal along the spatial axis depends on mating success, such that males who experience less success exhibit higher movement rates. In this case, the spatial location of the interface between the emerging species depends on the initial conditions. Importantly, however, the patterns of spatial segregation between the two marker-preference pairs reported by Payne and Krakauer (1997) turn out to be transient (de Cara and Dieckmann, unpublished).

In both of these cases of parapatric diversification, sexual selection causes different runaway processes in different spatial locations, through spatial variation either in viability selection or in the initial conditions. The analysis of these models illustrates that in most models of parapatric speciation ecological contact between the incipient species, which leads to genetic mixing, is a hindrance to the emergence of spatially segregated species. The results of Payne and Krakauer (1997) show that a viability gradient can, nevertheless, stabilize divergent Fisherian runaway processes in different spatial domains.

\section{Concluding Comments}

In this chapter we describe a theory of adaptive parapatric speciation that links ecological processes driving local divergence to global patterns of species abundance. In many traditional models of parapatric speciation it is assumed that some form of geographic differentiation is induced by discontinuities in the external environment, and that speciation is driven by divergent local adaptation or genetic drift in spatially distant locations and is hindered by gene flow because of ecological contact (Gavrilets 1999; Turelli et al. 2001; Chapter 6 in Dieckmann et al. 2004). This applies as much to models of parapatric speciation driven by sexual selection (Box 5) as to those driven by natural selection based on ecologically relevant traits. By contrast, the models described in this chapter focus on the adaptive processes that can generate local divergence in spatially extended, but genetically and environmentally continuous, populations. Ecological contact is a critical prerequisite for the operation of this alternative mechanism of parapatric speciation.

Our results show that local ecological contact may, in fact, be the driving force for parapatric speciation. In our models, gene flow is, of course, still a hindrance to local divergence, but the mechanisms that generate local disruptive selection require ecological contact. Local disruptiveness, in turn, selects for assortative mating, which reduces and eventually eliminates gene flow between the emerging species. The latter process is akin to reinforcement, but for the fact that in our models selection for prezygotic isolation emerges dynamically from frequency-dependent ecological interactions, as opposed to being the consequence of secondary contact. Evolution- 
ary branching in spatially structured sexual populations shows how adaptive speciation can result in spatial segregation between the emerging species, and thus suggests an answer to Endler's (1977) old question of how sharp geographic differentiation can evolve in a single, spatially and genetically continuous species despite the presence of gene flow and in the absence of abrupt environmental changes.

The dynamic and continuous unfolding of local processes into a global pattern, as observed in our models, indicates that the classic allopatry-sympatry controversy about speciation may often be ill-posed: what, in the end, results in an allopatric pattern of species abundance can be generated by an intrinsically sympatric evolutionary process. Inferring past processes from extant patterns is always difficult, and to understand speciation it is particularly important to distinguish between the two. This also applies to the study of hybrid zones, which are usually thought of as originating from secondary contact between species that were formed in allopatry (Barton and Hewitt 1989). Our results reinforce the question of whether many of these zones are, in fact, primary and have arisen as stepped phenotypic and genetic clines out of genetically continuous ancestral populations by the processes described in this chapter. That such processes can be an important agent for generating adaptive splits into abutting sister species may thus provide new perspectives on old problems (Doebeli and Dieckmann 2003; Tautz 2003) - perspectives that are supported by a number of recent empirical studies, such as on intertidal snails (Wilding et al. 2001) and on Anolis lizards (Thorpe and Richard 2001; Ogden and Thorpe 2002; see Chapter 16 in Dieckmann et al. 2004).

We conclude that spatial structure can facilitate speciation because local adaptation along an environmental gradient increases the degree of frequency dependence in spatially localized ecological interactions, and hence the likelihood that these interactions generate disruptive selection and evolutionary branching. With local adaptation and sufficiently low levels of mobility, short interaction distances imply that individuals interact more often with other individuals of similar phenotypes, which results in an increased negative frequency dependence of their fitness on similar phenotypes. This is a potentially important mechanism for generating divergence that seems to have been overlooked in past work on parapatric speciation. Interestingly, this mechanism implies that the degree of frequency dependence induced by spatial structure actually decreases for very steep environmental gradients, because in this case very different locally adapted phenotypes occur in close spatial proximity, so that dissimilar phenotypes compete even if their interactions are spatially localized. As a consequence, facilitation of evolutionary branching is most pronounced for environmental gradients of intermediate slope, a result that is fundamentally different from those expected in classic scenarios of parapatric speciation along linear gradients (e.g., Endler 1977; see also Box 4). Studying such spatial facilitation of adaptive divergence may even shed new light on the problem of species-area relationships, because this mechanism operates more effectively in larger spatial areas, and thus provides an intrinsic explanation for higher speciation rates in larger areas. In sum, as anticipated in classic speciation theories, geographic structure may, indeed, play an essential role in the generation of diversity, but its importance and the role of spatially gradual environmental change may only be appreciated fully when adaptive processes of divergence through spatially localized and frequency-dependent ecological interactions are taken into account.

\section{References}

Abrams PA, Matsuda H \& Harada Y (1993a). Evolutionary unstable fitness maxima and stable fitness minima of continuous traits. Evolutionary Ecology 7:465-487

Barraclough TG \& Vogler AP (2000). Detecting the geographical pattern of speciation from species-level phylogenies. The American Naturalist 155:419-434 
Barton NH (1999). Clines in polygenic traits. Genetical Research (Cambridge) 74:223-236

Barton NH \& Hewitt GM (1989). Adaptation, speciation, and hybrid zones. Nature 341:497-503

Case TJ \& Taper ML (2000). Interspecific competition, environmental gradients, gene flow, and the coevolution of species' borders. The American Naturalist 155:583-605

Christiansen FB (1991). On conditions for evolutionary stability for a continuously varying character. The American Naturalist 138:37-50

Day T (2000). Competition and the effect of spatial resource heterogeneity on evolutionary diversification. The American Naturalist 155:790-803

De Cara MAR \& Dieckmann U. Speciation by pattern formation revisited. Unpublished

Dieckmann U \& Law R (1996). The dynamical theory of coevolution: Derivation from stochastic ecological processes. Journal of Mathematical Biology 34:579-612

Dieckmann U \& Doebeli M (1999). On the origin of species by sympatric speciation. Nature 400:354-357

Dieckmann U, Doebeli M, Metz JAJ \& Tautz D (2004). Adaptive Speciation. Cambridge, UK: Cambridge University Press.

Dieckmann U, Law R \& Metz JAJ (2000). The Geometry of Ecological Interactions: Simplifying Spatial Complexity. Cambridge, UK: Cambridge University Press

Doebeli M \& Dieckmann U (2000). Evolutionary branching caused by different types of ecological interactions. The American Naturalist 156:S77-S101

Doebeli M \& Dieckmann U (2003). Speciation along environmental gradients. Nature 421:259263

Endler JA (1977). Geographic Variation, Speciation, and Clines. Princeton, NJ, USA: Princeton University Press

Eshel I (1983). Evolutionary and continuous stability. Journal of Theoretical Biology 103:99-111

Gavrilets S (1999). A dynamical theory of speciation on holey adaptive landscapes. The American Naturalist 154:1-22

Geritz SAH, Kisdi É, Meszéna G \& Metz JAJ (1998). Evolutionarily singular strategies and the adaptive growth and branching of the evolutionary tree. Evolutionary Ecology 12:35-57

Kirkpatrick M \& Barton NH (1997). Evolution of a species' range. The American Naturalist 150:1-23

Kirkpatrick M \& Servedio MR (1999). The reinforcement of mating preferences on an island. Genetics 151:865-884

Lande R (1979b). Quantitative genetic analysis of multivariate evolution, applied to brain:body size allometry. Evolution 33:402-416

Lande R (1982). Rapid origin of sexual isolation and character divergence in a cline. Evolution 36:213-223

Liou LW \& Price TD (1994). Speciation by reinforcement of premating isolation. Evolution 48:1451-1459

Losos JB \& Schluter D (2000). Analysis of an evolutionary species-area relationship. Nature 408:847-850

MacArthur RH \& Wilson EO (1967). Theory of Island Biogeography. Princeton, NJ, USA: Princeton University Press

Malhotra A \& Thorpe RS (1997c). Size and shape variation in a Lesser Antillean anole Anolis oculatus (Sauria: Iguanidae) in relation to habtitat. Biological Journal of the Linnean Society 60:53-72

Metz JAJ, Geritz SAH, Meszéna G, Jacobs FJA \& Van Heerwaarden JS (1996). Adaptive dynamics: A geometrical study of the consequences of nearly faithful reproduction. In Stochastic and Spatial Structures of Dynamical Systems, eds. Van Strien SJ \& Verduyn Lunel SM, pp. 183-231. Amsterdam, Netherlands: North Holland 
Mizera F \& Meszéna G (2003). Spatial niche packing, character displacement, and adaptive speciation along an environmental gradient. Evolutionary Ecology Research 5:363-382

Noor MA (1995). Speciation by natural selection in Drosophila. Nature 375:674-675

Ogden R \& Thorpe RS (2002). Molecular evidence for ecological speciation in tropical habitats. Proceedings of the National Academy of Sciences of the USA 99:13612-13615

Payne RJH \& Krakauer DC (1997). Sexual selection, space, and speciation. Evolution 51:1-9

Rosenzweig ML (1995). Species Diversity in Time and Space. Cambridge, UK: Cambridge University Press

Roughgarden J (1995). Anolis Lizards of the Caribbean. Oxford, UK: Oxford University Press

Servedio MR (2000). Reinforcement and the genetics of nonrandom mating. Evolution 54:21-29

Slatkin M (1978). Spatial patterns in the distribution of polygenic characters. Journal of Theoretical Biology 70:213-228

Tautz D (2003). Evolutionary biology: Splitting in space. Nature 421:225-226

Thorpe RS \& Richard M (2001). Evidence that ultraviolet markings are associated with patterns of molecular gene flow. Proceedings of the National Academy of Sciences of the USA 98:39293934

Turelli M, Barton NH \& Coyne JA (2001). Theory and speciation. Trends in Ecology and Evolution 16:330-343

Wilding CS, Grahame J \& Mill PJ (2001). Correlation of morphological diversity with molecular marker diversity in the rough periwinkle Littorina saxatilis (Olivi). Journal of Shellfish Research 20:501-508 